Commun. Fac. Sci. Univ. Ank. Ser. A1 Math. Stat.

Volume 69, Number 1, Pages 73-87(2020)

DOI: $10.31801 /$ cfsuasmas.508702

ISSN 1303-5991 E-ISSN 2618-6470

http://communications.science.ankara.edu.tr/index.php?series=A1

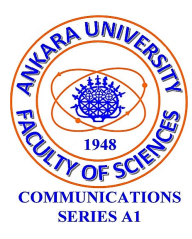

\title{
GENERALIZED FRACTIONAL MAXIMAL OPERATOR ON GENERALIZED LOCAL MORREY SPACES
}

\author{
A. KUCUKASLAN, V.S. GULIYEV, AND A. SERBETCI
}

\begin{abstract}
In this paper, we study the boundedness of generalized fractional maximal operator $M_{\rho}$ on generalized local Morrey spaces $L M_{p, \varphi}^{\left\{x_{0}\right\}}$ and generalized Morrey spaces $M_{p, \varphi}$, including weak estimates. Firstly, we prove the Spanne type boundedness of $M_{\rho}$ from the space $L M_{p, \varphi_{1}}^{\left\{x_{0}\right\}}$ to another $L M_{q, \varphi_{2}}^{\left\{x_{0}\right\}}$, $1<p<q<\infty$ and from $L M_{1, \varphi_{1}}^{\left\{x_{0}\right\}}$ to the weak space $W L M_{q, \varphi_{2}}^{\left\{x_{0}\right\}}$ for $p=1$ and $1<q<\infty$. Secondly, we prove the Adams type boundedness of $M_{\rho}$ from the space $M_{p, \varphi^{\frac{1}{p}}}$ to another $M_{q, \varphi^{\frac{1}{q}}}$ for $1<p<q<\infty$ and from $M_{1, \varphi}$ to the weak

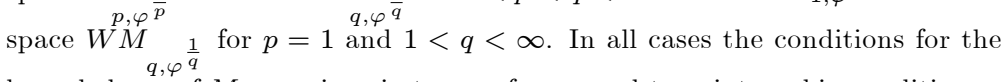
boundedness of $M_{\rho}$ are given in terms of supremal-type integral inequalities on $\left(\varphi_{1}, \varphi_{2}, \rho\right)$ and $(\varphi, \rho)$, which do not assume any assumption on monotonicity of $\varphi_{1}(x, r), \varphi_{2}(x, r)$ and $\varphi(x, r)$ in $r$.
\end{abstract}

\section{INTRODUCTION}

The classical Morrey spaces $M_{p, \lambda}$ were first introduced by Morrey in [21] to study the local behavior of solutions to second order elliptic partial differential equations. The generalized Morrey spaces $M_{p, \varphi}$ are obtained by replacing $r^{\lambda}$ in the definition of the Morrey space. During the last decades various classical operators, such as maximal, singular and potential operators were widely investigated in both in classical, generalized Morrey spaces and generalized local Morrey spaces. For the boundedness of the Hardy-Littlewood maximal operator, the fractional integral operator and the Calderón-Zygmund singular integral operators on these spaces, we refer the readers to [1, 9, 15, 16, 20, 22,

Received by the editors: January 07, 2019, Accepted: August 09, 2019.

2010 Mathematics Subject Classification. 42B20, 42B25, 42B35.

Key words and phrases. Generalized fractional maximal operator, generalized local Morrey spaces, generalized Morrey spaces.

The research of A. Kucukaslan was totally supported by the grant of The Scientific and Technological Research Council of Turkey (TUBITAK), [Grant-1059B191600675].

The research of V.S. Guliyev was partially supported by the Ministry of Education and Science of the Russian Federation (the Agreement number No. 02.a03.21.0008). C2020 Ankara University
Communications Faculty of Sciences University of Ankara-Series A1 Mathematics and Statistics 
For a measurable function $\rho:(0, \infty) \rightarrow(0, \infty)$ the generalized fractional maximal operator $M_{\rho}$ and the generalized fractional integral operator $I_{\rho}$ are defined by

$$
\begin{gathered}
M_{\rho} f(x)=\sup _{t>0} \frac{\rho(t)}{t^{n}} \int_{B(x, t)}|f(y)| d y, \\
I_{\rho} f(x)=\int_{\mathbb{R}^{n}} \frac{\rho(|x-y|)}{|x-y|^{n}} f(y) d y
\end{gathered}
$$

for any suitable function $f$ on $\mathbb{R}^{n}$. If $\rho(t) \equiv t^{\alpha}$, then $M_{\alpha} \equiv M_{t^{\alpha}}$ is the fractional maximal operator and $I_{\alpha} \equiv I_{t^{\alpha}}$ is the Riesz potential.

Spanne [24] and Adams [1] studied boundedness of the Riesz potential in Morrey spaces. Their results can be summarized as follows.

Theorem A. (Spanne, but published by Peetre [24]) Let $0<\alpha<n, 1<p<\frac{n}{\alpha}$, $0<\lambda<n-\alpha p$. Moreover, let $\frac{1}{p}-\frac{1}{q}=\frac{\alpha}{n}$ and $\frac{\lambda}{p}=\frac{\mu}{q}$. Then for $p>1$, the operator $I_{\alpha}$ is bounded from $M_{p, \lambda}$ to $M_{q, \mu}$ and for $p=1, I_{\alpha}$ is bounded from $M_{1, \lambda}$ to $W M_{q, \mu}$.

Theorem B. (Adams [1]) Let $0<\alpha<n, 1<p<\frac{n}{\alpha}, 0<\lambda<n-\alpha p$ and $\frac{1}{p}-\frac{1}{q}=\frac{\alpha}{n-\lambda}$. Then for $p>1$, the operator $I_{\alpha}$ is bounded from $M_{p, \lambda}$ to $M_{q, \lambda}$ and for $p=1, I_{\alpha}$ is bounded from $M_{1, \lambda}$ to $W M_{q, \lambda}$.

Nakai 22] proved the boundedness of the operators $I_{\rho}$ and $M_{\rho}$ from the generalized Morrey spaces $M_{p, \varphi_{1}}$ to the spaces $M_{q, \varphi_{2}}$ for suitable functions $\varphi_{1}$ and $\varphi_{2}$. The boundedness of $M_{\rho}$ and $I_{\rho}$ from the generalized Morrey spaces $M_{p, \varphi_{1}}$ to the spaces $M_{q, \varphi_{2}}$ is studied by Nakai [23], Eridani [10, Gunawan [18, Eridani, Gunawan and Nakai [12, Sawano, Sugano, Tanaka [25, Eridani, Gunawan, Nakai, Sawano [11, Guliyev, Ismayilova, Kucukaslan, Serbetci [17, Kucukaslan, Hasanov, Aykol [19].

In particular, the following statement containing both Theorem A and Theorem B was proved in [3, 4.

Theorem C. (3, 4) Let $1 \leq p<q<\infty, 0<\lambda, \mu<n$ and

$$
0<\alpha=\frac{n-\lambda}{p}-\frac{n-\mu}{q}<\frac{n}{p}
$$

Then, for $p>1$, the operator $I_{\alpha}$ is bounded from $M_{p, \lambda}$ to $M_{q, \mu}$, and, for $p=1, I_{\alpha}$ is bounded from $M_{1, \lambda}$ to $W M_{q, \mu}$.

In [3, 4] it was also proved that, under the assumptions of Theorem $\mathrm{C}$, the operator $I_{\alpha}$, for $p>1$, is bounded from the local Morrey space $L M_{p, \lambda}^{\left\{x_{0}\right\}}$ to $L M_{q, \mu}^{\left\{x_{0}\right\}}$, and, for $p=1$ from $L M_{1, \lambda}^{\left\{x_{0}\right\}}$ to the weak local Morrey space $W L M_{q, \mu}^{\left\{x_{0}\right\}}$.

Since, for some $c>0,\left(M_{\alpha} f\right)(x) \leq c\left(I_{\alpha}(|f|)\right)(x), x \in \mathbb{R}^{n}$, it follows that in Theorems A, B, C the operator $I_{\alpha}$ can be replaced by the operator $M_{\alpha}$ (including 
also the case $p=q$ ). For the operator $M_{\alpha}$ Theorem $\mathrm{C}$ was, in fact, earlier proved in $[5,6]$.

Guliyev [14] proved the Spanne and Adams type boundedness of $I_{\alpha}$ from the spaces $M_{p, \varphi_{1}}\left(\mathbb{R}^{n}\right)$ to $M_{q, \varphi_{2}}\left(\mathbb{R}^{n}\right)$ without any assumption on monotonicity of $\varphi_{1}$, $\varphi_{2}$. Paper [7] should be mentioned where for $\alpha=n\left(\frac{1}{p}-\frac{1}{q}\right)$ necessary and sufficient conditions of $\varphi_{1}$ and $\varphi_{2}$ are obtained. In [17, by using the method given in [13, the Spanne and Adams type boundedness of the operator $I_{\rho}$ from the generalized local Morrey space $L M_{p, \varphi_{1}}^{\left\{x_{0}\right\}}$ to another one $L M_{q, \varphi_{2}}^{\left\{x_{0}\right\}}$ were proved.

The main goal of this paper is to show that the boundedness of the generalized fractional maximal operator $M_{\rho}$ in generalized local Morrey spaces $L M_{p, \varphi}^{\left\{x_{0}\right\}}$ and generalized Morrey spaces $M_{p, \varphi}$ can be obtained under weaker assumptions on $\rho$, namely in terms of the so-called supremal operators. More precisely, we find sufficient conditions, in supremal terms, on the functions $\left(\varphi_{1}, \varphi_{2}, \rho\right)$ which ensure the boundedness of the operator $M_{\rho}$ from one generalized local Morrey space $L M_{p, \varphi_{1}}^{\left\{x_{0}\right\}}$ to another $L M_{q, \varphi_{2}}^{\left\{x_{0}\right\}}$ for $1<p<q<\infty$ and from $L M_{1, \varphi_{1}}^{\left\{x_{0}\right\}}$ to the weak space $W L M_{q, \varphi_{2}}^{\left\{x_{0}\right\}}$ for $p=1$ and $1<q<\infty$. We also find conditions on the pair $(\varphi, \rho)$ which ensure the Adams type boundedness of $M_{\rho}$ from the spaces $M_{p, \varphi^{\frac{1}{p}}}$ to another $M_{q, \varphi^{\frac{1}{q}}}$ for $1<p<q<\infty$ and from $M_{1, \varphi}$ to the weak space $W M_{q, \varphi^{\frac{1}{q}}}^{p, \varphi^{\frac{1}{p}}}$ for $p=1$ and $1<q<\infty$.

By $A \lesssim B$ we mean that $A \leq C B$ with some positive constant $C$ independent of appropriate quantities. If $A \lesssim B$ and $B \lesssim A$, we write $A \approx B$ and say that $A$ and $B$ are equivalent.

\section{Preliminaries}

For $x \in \mathbb{R}^{n}$ and $r>0$, we denote by $B(x, r)$ the open ball centered at $x$ of radius $r$, and by ${ }^{\complement} B(x, r)$ denote its complement. Let $|B(x, r)|$ be the Lebesgue measure of the ball $B(x, r)$. Therefore $|B(x, r)|=w_{n} r^{n}$, where $w_{n}$ denotes the volume of the unit ball in $\mathbb{R}^{n}$.

Definition 2.1. Let $\varphi(x, r)$ be a positive measurable function on $\mathbb{R}^{n} \times(0, \infty)$ and $1 \leq p<\infty$. We denote by $M_{p, \varphi} \equiv M_{p, \varphi}\left(\mathbb{R}^{n}\right)$ the generalized Morrey space, the space of all functions $f \in L_{p}^{\text {loc }}\left(\mathbb{R}^{n}\right)$ with finite norm

$$
\|f\|_{M_{p, \varphi}}=\sup _{x \in \mathbb{R}^{n}, r>0} \varphi(x, r)^{-1}|B(x, r)|^{-\frac{1}{p}}\|f\|_{L_{p}(B(x, r))} .
$$

Also by $W M_{p, \varphi} \equiv W M_{p, \varphi}\left(\mathbb{R}^{n}\right)$ we denote the weak generalized Morrey space of all functions $f \in W L_{p}^{\text {loc }}\left(\mathbb{R}^{n}\right)$ for which

$$
\|f\|_{W M_{p, \varphi}}=\sup _{x \in \mathbb{R}^{n}, r>0} \varphi(x, r)^{-1}|B(x, r)|^{-\frac{1}{p}}\|f\|_{W L_{p}(B(x, r))}<\infty .
$$


According to this definition, we recover the Morrey space $M_{p, \lambda}$, the weak Morrey space $W M_{p, \lambda}$ respectively, under the choice $\varphi(x, r)=r^{\frac{\lambda-n}{p}}$ :

$$
M_{p, \lambda}=\left.M_{p, \varphi}\right|_{\varphi(x, r)=r} \frac{\lambda-n}{p}, W M_{p, \lambda}=\left.W M_{p, \varphi}\right|_{\varphi(x, r)=r} \frac{\lambda-n}{p} .
$$

Definition 2.2. Let $\varphi(x, r)$ be a positive measurable function on $\mathbb{R}^{n} \times(0, \infty)$ and $1 \leq p<\infty$. We denote by $L M_{p, \varphi} \equiv L M_{p, \varphi}\left(\mathbb{R}^{n}\right)$ the generalized local (central) Morrey space, the space of all functions $f \in L_{p}^{\text {loc }}\left(\mathbb{R}^{n}\right)$ with finite norm

$$
\|f\|_{L M_{p, \varphi}}=\sup _{r>0} \varphi(0, r)^{-1}|B(0, r)|^{-\frac{1}{p}}\|f\|_{L_{p}(B(0, r))} .
$$

Also by $W L M_{p, \varphi} \equiv W L M_{p, \varphi}\left(\mathbb{R}^{n}\right)$ we denote the weak generalized local (central) Morrey space of all functions $f \in W L_{p}^{\text {loc }}\left(\mathbb{R}^{n}\right)$ for which

$$
\|f\|_{W L M_{p, \varphi}}=\sup _{r>0} \varphi(0, r)^{-1}|B(0, r)|^{-\frac{1}{p}}\|f\|_{W L_{p}(B(0, r))}<\infty .
$$

Definition 2.3. Let $\varphi(x, r)$ be a positive measurable function on $\mathbb{R}^{n} \times(0, \infty)$ and $1 \leq p<\infty$. For any fixed $x_{0} \in \mathbb{R}^{n}$ we denote by $L M_{p, \varphi}^{\left\{x_{0}\right\}} \equiv \operatorname{LM}_{p, \varphi}^{\left\{x_{0}\right\}}\left(\mathbb{R}^{n}\right)$ the generalized local Morrey space, the space of all functions $f \in L_{p}^{\text {loc }}\left(\mathbb{R}^{n}\right)$ with finite norm

$$
\|f\|_{L M_{p, \varphi}^{\left\{x_{0}\right\}}}=\left\|f\left(x_{0}+\cdot\right)\right\|_{L M_{p, \varphi}} .
$$

Also by $W L M_{p, \varphi}^{\left\{x_{0}\right\}} \equiv W L M_{p, \varphi}^{\left\{x_{0}\right\}}\left(\mathbb{R}^{n}\right)$ we denote the weak generalized local Morrey space of all functions $f \in W L_{p}^{\text {loc }}\left(\mathbb{R}^{n}\right)$ for which

$$
\|f\|_{W L M_{p, \varphi}^{\left\{x_{0}\right\}}}=\left\|f\left(x_{0}+\cdot\right)\right\|_{W L M_{p, \varphi}}<\infty .
$$

According to this definition, we recover the local Morrey space $L M_{p, \lambda}^{\left\{x_{0}\right\}}$ and weak local Morrey space $W L M_{p, \lambda}^{\left\{x_{0}\right\}}$ under the choice $\varphi\left(x_{0}, r\right)=r^{\frac{\lambda-n}{p}}$ :

$$
L M_{p, \lambda}^{\left\{x_{0}\right\}}=\left.L M_{p, \varphi}^{\left\{x_{0}\right\}}\right|_{\varphi\left(x_{0}, r\right)=r} \frac{\lambda-n}{p}, W L M_{p, \lambda}^{\left\{x_{0}\right\}}=\left.W L M_{p, \varphi}^{\left\{x_{0}\right\}}\right|_{\varphi\left(x_{0}, r\right)=r} \frac{\lambda-n}{p} .
$$

Definition 2.4. Let $\mathfrak{M}(0, \infty)$ be the set of all Lebesgue-measurable functions on $(0, \infty)$ and $\mathfrak{M}^{+}(0, \infty)$ its subset consisting of all non-negative functions on $(0, \infty)$. We define a cone $\mathbb{A}$ by the set of the functions $\varphi \in \mathfrak{M}^{+}(0, \infty)$ which are nondecreasing on $(0, \infty)$ and such that $\lim _{t \rightarrow 0+} \varphi(t)=0$, briefly

$$
\mathbb{A}=\left\{\varphi \in \mathfrak{M}^{+}(0, \infty ; \uparrow): \lim _{t \rightarrow 0+} \varphi(t)=0\right\}
$$

Definition 2.5. [8] Let $u$ be a continuous and non-negative function on $(0, \infty)$. We define the supremal operator $\bar{S}_{u}$ on $g \in \mathfrak{M}(0, \infty)$ by

$$
\left(\bar{S}_{u} g\right)(r):=\|u(t) g(t)\|_{L_{\infty}(r, \infty)}, r \in(0, \infty) .
$$


Let $v$ be a non-negative measurable function on $(0, \infty)$. We denote by $L_{\infty, v}(0, \infty)$ the space of all functions $g(t), t>0$ with finite norm

$$
\|g\|_{L_{\infty, v}(0, \infty)}=\sup _{t>0} v(t) g(t)
$$

and $L_{\infty}(0, \infty) \equiv L_{\infty, 1}(0, \infty)$. The following lemma is proved analogously to Lemma 5.2 in 8 .

Lemma 2.1. 8] Let $v_{1}$ and $v_{2}$ be weights and $0<\left\|v_{1}\right\|_{L_{\infty}(t, \infty)}<\infty$ for any $t>0$ and let $u$ be a continuous non-negative function on $(0, \infty)$. Then the operator $\bar{S}_{u}$ is bounded from $L_{\infty, v_{1}}(0, \infty)$ to $L_{\infty, v_{2}}(0, \infty)$ on the cone $\mathbb{A}$ if and only if

$$
\left\|v_{2} \bar{S}_{u}\left(\left\|v_{1}\right\|_{L_{\infty}(\cdot, \infty)}^{-1}\right)\right\|_{L_{\infty}(0, \infty)}<\infty
$$

The following lemma was proved in [17].

Lemma 2.2. 17. Let $v_{1}, v_{2}$ be non-negative measurable functions satisfying $0<$ $\left\|v_{1}\right\|_{L_{\infty}(t, \infty)}<\infty$ for any $t>0$. Then the identity operator $I$ is bounded from $L_{\infty, v_{1}}(0, \infty)$ to $L_{\infty, v_{2}}(0, \infty)$ on the cone $\mathbb{A}$ if and only if

$$
\left\|v_{2}\left(\left\|v_{1}\right\|_{L_{\infty}(\cdot, \infty)}^{-1}\right)\right\|_{L_{\infty}(0, \infty)}<\infty
$$

3. Spanne type Result For the operator $M_{\rho}$ IN the SPACes $L M_{p, \varphi}^{\left\{x_{0}\right\}}$

We assume that

$$
\sup _{1 \leq t<\infty} \frac{\rho(t)}{t^{n}}<\infty
$$

so that the fractional maximal functions $M_{\rho} f$ are well defined, at least for characteristic functions $1 /|x|^{2 n}$ of complementary balls:

$$
f(x)=\frac{\chi_{\mathbb{R}^{n} \backslash B(0,1)}(x)}{|x|^{2 n}} .
$$

In addition, we shall also assume that $\rho$ satisfies the growth condition: there exist constants $C_{1}>0$ and $0<2 k_{1}<k_{2}<\infty$ such that

$$
\sup _{r<s \leq 2 r} \frac{\rho(s)}{s^{n}} \leq C_{1} \sup _{k_{1} r<t<k_{2} r} \frac{\rho(t)}{t^{n}}, r>0 .
$$

This condition is weaker than the usual doubling condition for the function $\frac{\rho(t)}{t^{n}}$ : there exists a constant $C_{2}>0$ such that

$$
\frac{1}{C_{2}} \frac{\rho(t)}{t^{n}} \leq \frac{\rho(r)}{r^{n}} \leq C_{2} \frac{\rho(t)}{t^{n}},
$$

whenever $r$ and $t$ satisfy $r, t>0$ and $\frac{1}{2} \leq \frac{r}{t} \leq 2$. 
Remark 3.1. Typical examples of $\rho(t)$ that we envisage are, for $0<\alpha<n$

and, for $c>0$

$$
\rho(t) \equiv\left\{\begin{array}{cc}
t^{\alpha} \log (e / t), & 0<t \leq 1 \\
\frac{t^{\alpha}}{\log (e t)}, & 1 \leq t<\infty
\end{array}\right.
$$

$$
\rho(t) \equiv\left\{\begin{array}{cc}
t^{\alpha}, & 0<t \leq 1 \\
e^{c} e^{-c t^{2}}, & 1 \leq t<\infty .
\end{array}\right.
$$

The second one is used to control the Bessel potential (see also [26]).

The boundedness of the operator $I_{\rho}$ in the spaces $L_{p}\left(\mathbb{R}^{n}\right)$ can be found in [11. Let $\frac{\rho(t)}{t^{n}}$ be almost decreasing, that is, there exists a constant $C$ such that $\frac{\rho(t)}{t^{n}} \leq$ $C \frac{\rho(s)}{s^{n}}$ for $s<t$. In this case we get

$$
\begin{aligned}
M_{\rho} f(x) & =\sup _{t>0} \frac{\rho(t)}{t^{n}} \int_{B(x, t)}|f(y)| d y \\
& \lesssim \sup _{t>0} \int_{B(x, t)} \frac{\rho(|x-y|)}{|x-y|^{n}}|f(y)| d y \\
& =\int_{\mathbb{R}^{n}} \frac{\rho(|x-y|)}{|x-y|^{n}}|f(y)| d y=I_{\rho}(|f|)(x) .
\end{aligned}
$$

For proving our main results, we need the following estimate.

Lemma 3.3. If $B_{0}:=B\left(x_{0}, r_{0}\right) \subset B(x, r)$ and $\rho$ satisfies the doubling condition. Then $\rho\left(r_{0}\right) \lesssim M_{\rho} \chi_{B_{0}}(x)$ for every $x \in B_{0}$.

Proof. Let $\rho$ satisfy the doubling condition, then

$$
\mathcal{M}_{\rho} f(x) \lesssim M_{\rho} f(x)
$$

where $\mathcal{M}_{\rho}(f)(x)=\sup _{B \ni x} \frac{\rho\left(r_{B}\right)}{|B|} \int_{B}|f(y)| d y$ and $r_{B}$ is the center of the ball $B$.

Now let $x \in B_{0}$. By using $(3.3)$, we get

$$
\begin{aligned}
M_{\rho} \chi_{B_{0}}(x) & \gtrsim \mathcal{M}_{\rho} \chi_{B_{0}}(x)=\sup _{B \ni x} \frac{\rho\left(r_{B}\right)}{|B|}\left|B \cap B_{0}\right| \\
& \gtrsim \frac{\rho\left(r_{0}\right)}{\left|B_{0}\right|}\left|B_{0} \cap B_{0}\right|=\rho\left(r_{0}\right) .
\end{aligned}
$$

The following lemma is valid.

Lemma 3.4. Let $1 \leq p<q<\infty$.

(1) The condition

$$
\rho(r) \leq C r^{\frac{n}{p}-\frac{n}{q}}
$$

for all $r>0$, where $C>0$ does not depend on $r$, is sufficient for the boundedness of $M_{\rho}$ from $L_{p}\left(\mathbb{R}^{n}\right)$ to $W L_{q}\left(\mathbb{R}^{n}\right)$. Moreover, if $p>1$, then the condition (3.4) is sufficient for the boundedness of $M_{\rho}$ from $L_{p}\left(\mathbb{R}^{n}\right)$ to $L_{q}\left(\mathbb{R}^{n}\right)$. 
(2) If $\rho$ satisfies the doubling condition, then the condition (3.4) is necessary for the boundedness of $M_{\rho}$ from $L_{p}\left(\mathbb{R}^{n}\right)$ to $W L_{q}\left(\mathbb{R}^{n}\right)$ and from $L_{p}\left(\mathbb{R}^{n}\right)$ to $L_{q}\left(\mathbb{R}^{n}\right)$ for $p>1$.

(3) If $\rho$ satisfies the doubling condition and the supremal regularity condition

$$
\sup _{r<t<\infty} \rho(t) t^{-\frac{n}{p}} \leq C \rho(r) r^{-\frac{n}{p}}
$$

holds for all $r>0$, where $C>0$ does not depend on $r$, then the condition (3.4) is necessary and sufficient for the boundedness of $M_{\rho}$ from $L_{p}\left(\mathbb{R}^{n}\right)$ to $W L_{q}\left(\mathbb{R}^{n}\right)$. Moreover, if $p>1$, then the condition (3.4) is necessary and sufficient for the boundedness of $M_{\rho}$ from $L_{p}\left(\mathbb{R}^{n}\right)$ to $L_{q}\left(\mathbb{R}^{n}\right)$.

Proof. (1) Suppose $\rho$ satisfies the condition 3.4. Then

$$
M_{\rho} f(x) \lesssim M_{\frac{n}{p}-\frac{n}{q}} f(x) .
$$

Since the operator $M_{\frac{n}{p}-\frac{n}{q}}$ is bounded from $L_{p}\left(\mathbb{R}^{n}\right)$ to $W L_{q}\left(\mathbb{R}^{n}\right)$ and for $p>1$ from $L_{p}\left(\mathbb{R}^{n}\right)$ to $L_{q}\left(\mathbb{R}^{n}\right)$, then from 3.5 we get the statement (1).

(2) Now we shall prove the second part. Let $B_{0}=B\left(x_{0}, r_{0}\right)$ and $x \in B_{0}$. By Lemma 3.3 . we have $\rho\left(r_{0}\right) \lesssim M_{\rho} \chi_{B_{0}}(x)$. Therefore, we have

$$
\begin{aligned}
& \rho\left(r_{0}\right) \lesssim r_{0}^{-\frac{n}{q}}\left\|M_{\rho} \chi_{B_{0}}\right\|_{W L_{q}\left(B_{0}\right)} \lesssim r_{0}^{-\frac{n}{q}}\left\|M_{\rho} \chi_{B_{0}}\right\|_{W L_{q}\left(\mathbb{R}^{n}\right)} \\
& \lesssim r_{0}^{-\frac{n}{q}}\left\|\chi_{B_{0}}\right\|_{L_{p}\left(\mathbb{R}^{n}\right)} \lesssim r_{0}^{\frac{n}{p}-\frac{n}{q}}
\end{aligned}
$$

and for $p>1$

$$
\begin{aligned}
\rho\left(r_{0}\right) & \lesssim r_{0}^{-\frac{n}{q}}\left\|M_{\rho} \chi_{B_{0}}\right\|_{L_{q}\left(B_{0}\right)} \underset{r_{0}^{-\frac{n}{q}}}{\lesssim}\left\|M_{\rho} \chi_{B_{0}}\right\|_{L_{q}\left(\mathbb{R}^{n}\right)} \\
& \lesssim r_{0}^{-\frac{n}{q}}\left\|\chi_{B_{0}}\right\|_{L_{p}\left(\mathbb{R}^{n}\right)} \lesssim r_{0}^{\frac{n}{p}-\frac{n}{q}}
\end{aligned}
$$

holds for every $r_{0}>0$, hence the proof of statement (2) is completed.

(3) From the first and second statements the third statement of the lemma follows.

The following lemma is valid.

Lemma 3.5. Let $1 \leq p<q<\infty$ and let $\rho(t)$ satisfy the conditions 3.1), 3.2 and (3.4). Then the inequality

$$
\left\|M_{\rho} f\right\|_{W L_{q}\left(B\left(x_{0}, r\right)\right)} \lesssim\|f\|_{L_{p}\left(B\left(x_{0}, 2 r\right)\right)}+r^{\frac{n}{q}} \sup _{t>r}\|f\|_{L_{p}\left(B\left(x_{0}, t\right)\right)} \frac{\rho(t)}{t^{\frac{n}{p}}}
$$

holds for any ball $B\left(x_{0}, r\right)$ and for all $f_{p}^{\text {loc }}\left(\mathbb{R}^{n}\right)$.

If $p>1$, then the inequality

$$
\left\|M_{\rho} f\right\|_{L_{q}\left(B\left(x_{0}, r\right)\right)} \lesssim\|f\|_{L_{p}\left(B\left(x_{0}, 2 r\right)\right)}+r^{\frac{n}{q}} \sup _{t>r}\|f\|_{L_{p}\left(B\left(x_{0}, t\right)\right)} \frac{\rho(t)}{t^{\frac{n}{p}}}
$$

holds for any ball $B\left(x_{0}, r\right)$ and for all $f_{p}^{\text {loc }}\left(\mathbb{R}^{n}\right)$. 
Proof. Let $1 \leq p<q<\infty$ and let $\rho(t)$ satisfy the conditions 3.1, 3.2) and 3.4. For arbitrary $x_{0} \in \mathbb{R}^{n}$, set $B=B\left(x_{0}, r\right)$ for the ball centered at $x_{0}$ and of radius $r$. Write $f=f_{1}+f_{2}$ with $f_{1}=f \chi_{2 B}$ and $f_{2}=f \chi_{{ }^{c}}(2 B)$. Hence

$$
\left\|M_{\rho} f\right\|_{W L_{q}(B)} \leq\left\|M_{\rho} f_{1}\right\|_{W L_{q}(B)}+\left\|M_{\rho} f_{2}\right\|_{W L_{q}(B)} .
$$

Since $f_{1} \in L_{p}\left(\mathbb{R}^{n}\right), M_{\rho} f_{1} \in W L_{q}\left(\mathbb{R}^{n}\right)$ and by Lemma $3.4 M_{\rho}$ is bounded from $L_{p}\left(\mathbb{R}^{n}\right)$ to $W L_{q}\left(\mathbb{R}^{n}\right)$. Thus it follows that

$$
\left\|M_{\rho} f_{1}\right\|_{W L_{q}(B)} \leq\left\|M_{\rho} f_{1}\right\|_{W L_{q}\left(\mathbb{R}^{n}\right)} \leq C\left\|f_{1}\right\|_{L_{p}\left(\mathbb{R}^{n}\right)}=C\|f\|_{L_{p}(2 B)},
$$

where constant $C>0$ is independent of $f$.

Let $x$ be an arbitrary point from $B$. If $B(x, t) \cap{ }^{\mathrm{C}}(2 B) \neq \emptyset$, then $t>r$. Indeed, if $y \in B(x, t) \cap{ }^{c}(2 B)$, then $t>|x-y| \geq\left|x_{0}-y\right|-\left|x_{0}-x\right|>2 r-r=r$.

On the other hand, $B(x, t) \cap{ }^{\mathrm{c}}(2 B) \subset B\left(x_{0}, 2 t\right)$. Indeed, $y \in B(x, t) \cap{ }^{\mathrm{c}}(2 B)$, then we get $\left|x_{0}-y\right| \leq|x-y|+\left|x_{0}-x\right|<t+r<2 t$.

Hence

$$
\begin{aligned}
M_{\rho} f_{2}(x) & =\sup _{t>0} \frac{\rho(t)}{t^{n}} \int_{B(x, t) \cap^{\complement}(2 B)}|f(y)| d y \\
& \lesssim \sup _{t>r} \frac{\rho(2 t)}{(2 t)^{n}} \int_{B\left(x_{0}, 2 t\right)}|f(y)| d y \\
& =\sup _{t>2 r} \frac{\rho(t)}{t^{n}} \int_{B\left(x_{0}, t\right)}|f(y)| d y
\end{aligned}
$$

Therefore, for all $x \in B$ we have

$$
M_{\rho} f_{2}(x) \lesssim \sup _{t>2 r} \frac{\rho(t)}{t^{n}} \int_{B\left(x_{0}, t\right)}|f(y)| d y
$$

Thus

$$
\begin{aligned}
\left\|M_{\rho} f\right\|_{W L_{q}(B)} \leq\left\|M_{\rho} f\right\|_{L_{q}(B)} & \lesssim\|f\|_{L_{p}(2 B)}+|B|^{\frac{1}{q}} \sup _{t>2 r}\|f\|_{L_{1}\left(B\left(x_{0}, t\right)\right)} \frac{\rho(t)}{t^{n}} \\
& \lesssim\|f\|_{L_{p}(2 B)}+r^{\frac{n}{q}} \sup _{t>2 r}\|f\|_{L_{p}\left(B\left(x_{0}, t\right)\right)} \frac{\rho(t)}{t^{\frac{n}{p}}} .
\end{aligned}
$$

Let $p>1$. From the $(p, q)$ boundedness of $M_{\rho}$ and $(3.4)$ it follows that:

$$
\left\|M_{\rho} f_{1}\right\|_{L_{q}(B)} \leq\left\|M_{\rho} f_{1}\right\|_{L_{q}\left(\mathbb{R}^{n}\right)} \lesssim\left\|f_{1}\right\|_{L_{p}\left(\mathbb{R}^{n}\right)}=\|f\|_{L_{p}(2 B)} .
$$

Then by (3.8) and (3.9) we get the inequality 3.6$)$.

The following theorem is one of the main results of the paper in which we get the Spanne type boundedness of the generalized fractional maximal operator $M_{\rho}$ in the generalized local Morrey spaces $L M_{p, \varphi}^{\left\{x_{0}\right\}}$. 
Theorem 3.1. Let $x_{0} \in \mathbb{R}^{n}, 1 \leq p<q<\infty$, and let the function $\rho$ satisfy the conditions (3.1), 3.2) and (3.4). Let also $\left(\varphi_{1}, \varphi_{2}\right)$ satisfy the conditions

$$
\begin{gathered}
\operatorname{essinf}_{t<s<\infty} \varphi_{1}\left(x_{0}, s\right) s^{\frac{n}{p}} \leq C \varphi_{2}\left(x_{0}, \frac{t}{2}\right) t^{\frac{n}{q}}, \\
\sup _{t>r}\left(\underset{t<s<\infty}{\operatorname{essinf}} \varphi_{1}\left(x_{0}, s\right) s^{\frac{n}{p}}\right) \frac{\rho(t)}{t^{\frac{n}{p}}} \leq C \varphi_{2}\left(x_{0}, r\right),
\end{gathered}
$$

where $C$ does not depend on $x_{0}$ and $r$. Then the operator $M_{\rho}$ is bounded from $L M_{p, \varphi_{1}}^{\left\{x_{0}\right\}}$ to $W L M_{q, \varphi_{2}}^{\left\{x_{0}\right\}}$ and for $p>1$ from $L M_{p, \varphi_{1}}^{\left\{x_{0}\right\}}$ to $L M_{q, \varphi_{2}}^{\left\{x_{0}\right\}}$. Moreover,

$$
\left\|M_{\rho} f\right\|_{W L M_{q, \varphi_{2}}^{\left\{x_{0}\right\}}} \lesssim\|f\|_{L M_{p, \varphi 1}^{\left\{x_{0}\right\}}}
$$

and for $p>1$

$$
\left\|M_{\rho} f\right\|_{L M_{q, \varphi_{2}}^{\left\{x_{0}\right\}}} \lesssim\|f\|_{L M_{p, \varphi_{1}}^{\left\{x_{0}\right\}}}
$$

Proof. Let the function $\rho$ satisfy the conditions 3.1$),(3.2),(3.4)$, and also $\left(\varphi_{1}, \varphi_{2}\right)$ satisfy the conditions (3.10) and 3.11). By Lemmas 2.1. 2.2 and 3.5 we have

$$
\begin{aligned}
\left\|M_{\rho} f\right\|_{W L M_{q, \varphi_{2}}^{\left\{x_{0}\right\}}} & \lesssim \sup _{r>0} \varphi_{2}\left(x_{0}, r\right)^{-1} r^{-\frac{n}{q}}\|f\|_{L_{p}\left(B\left(x_{0}, 2 r\right)\right)} \\
& +\sup _{r>0} \varphi_{2}\left(x_{0}, r\right)^{-1} \sup _{t>r}\|f\|_{L_{p}\left(B\left(x_{0}, t\right)\right)} \frac{\rho(t)}{t^{\frac{n}{p}}} \\
& \approx \sup _{r>0} \varphi_{1}\left(x_{0}, r\right)^{-1} r^{-\frac{n}{p}}\|f\|_{L_{p}\left(B\left(x_{0}, r\right)\right)}=\|f\|_{L M_{p, \varphi_{1}}^{\left\{x_{0}\right\}}}
\end{aligned}
$$

and for $p>1$

$$
\begin{aligned}
\left\|M_{\rho} f\right\|_{L M_{q, \varphi_{2}}^{\left\{x_{0}\right\}}} & \lesssim \sup _{r>0} \varphi_{2}\left(x_{0}, r\right)^{-1} r^{-\frac{n}{q}}\|f\|_{L_{p}\left(B\left(x_{0}, 2 r\right)\right)} \\
& +\sup _{r>0} \varphi_{2}\left(x_{0}, r\right)^{-1} \sup _{t>r}\|f\|_{L_{p}\left(B\left(x_{0}, t\right)\right)} \frac{\rho(t)}{t^{\frac{n}{p}}} \\
& \approx \sup _{r>0} \varphi_{1}\left(x_{0}, r\right)^{-1} r^{-\frac{n}{p}}\|f\|_{L_{p}\left(B\left(x_{0}, r\right)\right)}=\|f\|_{L M_{p, \varphi 1}^{\left\{x_{0}\right\}}} .
\end{aligned}
$$

In the following corollary we get the boundedness of the generalized fractional maximal operator $M_{\rho}$ on generalized Morrey spaces $M_{p, \varphi}$.

Corollary 3.1. Let $1 \leq p<q<\infty$, the function $\rho$ satisfy the conditions (3.1), (3.2) and (3.4). Let also $\left(\varphi_{1}, \varphi_{2}\right)$ satisfy the following conditions

$$
\begin{gathered}
\operatorname{essinf}_{r<t<\infty} \varphi_{1}(x, t) t^{\frac{n}{p}} \leq C \varphi_{2}\left(x, \frac{r}{2}\right) r^{\frac{n}{q}}, \\
\sup _{t>r}\left(\underset{t<s<\infty}{\operatorname{essinf}} \varphi_{1}(x, s) s^{\frac{n}{p}}\right) \frac{\rho(t)}{t^{\frac{n}{p}}} \leq C \varphi_{2}(x, r),
\end{gathered}
$$

where $C$ does not depend on $x$ and $r$. Then the operator $M_{\rho}$ is bounded from $M_{p, \varphi_{1}}$ to $W M_{q, \varphi_{2}}$ and for $p>1$ from $M_{p, \varphi_{1}}$ to $M_{q, \varphi_{2}}$. 
In the case $\rho(t)=t^{\alpha}$ from Theorem 3.1 we get new Spanne type result for fractional maximal operator $M_{\alpha}$ on generalized local Morrey spaces.

Corollary 3.2. Let $x_{0} \in \mathbb{R}^{n}, 0<\alpha<n, 1 \leq p<q<\infty$ and $1 / p-1 / q=\alpha / n$. Let also $\left(\varphi_{1}, \varphi_{2}\right)$ satisfy the condition

$$
\sup _{t>r}\left(\underset{t<s<\infty}{\operatorname{essinf}} \varphi_{1}\left(x_{0}, s\right) s^{\frac{n}{p}}\right) t^{-\frac{n}{q}} \leq C \varphi_{2}\left(x_{0}, r\right)
$$

where $C$ does not depend on $r$. Then the operator $M_{\alpha}$ is bounded from $L M_{p, \varphi_{1}}^{\left\{x_{0}\right\}}$ to $L M_{q, \varphi_{2}}^{\left\{x_{0}\right\}}$ for $p>1$ and from $L M_{1, \varphi_{1}}^{\left\{x_{0}\right\}}$ to $W L M_{q, \varphi_{2}}^{\left\{x_{0}\right\}}$ for $p=1$.

Also in the case $\rho(t)=t^{\alpha}$ and $\varphi(x, t)=t^{\frac{\lambda-n}{p}}, 0<\lambda<n$ from Theorem 3.1 we get local Morrey space variant of Theorem A.

Corollary 3.3. Let $x_{0} \in \mathbb{R}^{n}, 0<\alpha<n, 1<p<\frac{n}{\alpha}, 0<\lambda<n-\alpha p$. Moreover, let $\alpha=\frac{n}{p}-\frac{n}{q}$ and $\frac{\lambda}{p}=\frac{\mu}{q}$. Then for $p>1$, the operator $M_{\alpha}$ is bounded from $L M_{p, \lambda}^{\left\{x_{0}\right\}}$ to $L M_{q, \mu}^{\left\{x_{0}\right\}}$ and for $p=1, M_{\alpha}$ is bounded from $L M_{1, \lambda}^{\left\{x_{0}\right\}}$ to $W L M_{q, \mu}^{\left\{x_{0}\right\}}$.

Remark 3.2. For this case $\alpha=\frac{n}{p}-\frac{n}{q}$ necessary and sufficient conditions for the boundedness of $I_{\alpha}$ from $M_{p, \varphi_{1}}$ to $M_{q, \varphi_{2}}$ are obtained in [4].

\section{Adams type Result for the operator $M_{\rho}$ IN the spaces $M_{p, \varphi}$}

The following theorem was proved in [2].

Theorem D. Let $1 \leq p<\infty$ and $\left(\varphi_{1}, \varphi_{2}\right)$ satisfy the condition

$$
\sup _{r<t<\infty} t^{-\frac{n}{p}} \operatorname{essinf}_{t<s<\infty} \varphi_{1}(x, s) s^{\frac{n}{p}} \leq C \varphi_{2}(x, r)
$$

where $C$ does not depend on $x$ and $r$. Then the operator $M$ is bounded from $M_{p, \varphi_{1}}$ to $W M_{p, \varphi_{2}}$ and for $p>1$, the operator $M$ is bounded from $M_{p, \varphi_{1}}$ to $M_{p, \varphi_{2}}$.

The following theorem is another main result of the paper, in which we get the Adams type boundedness of the generalized fractional maximal operator $M_{\rho}$ in the generalized Morrey spaces $M_{p, \varphi}$.

Theorem 4.2. Let $1 \leq p<q<\infty, \frac{\rho(t)}{t^{n}}$ be almost decreasing, and let $\rho(t)$ satisfy the condition (3.2) and the inequality

$$
\int_{0}^{k_{2} r} \frac{\rho(s)}{s} d s \leq C \rho(r),
$$

where $k_{2}$ is given by the condition 3.2 and $C$ does not depend on $r>0$. Let also $\varphi(x, t)$ satisfy the conditions

$$
\sup _{r<t<\infty} t^{-n} \operatorname{essinf}_{t<s<\infty} \varphi(x, s) s^{n} \leq C \varphi(x, r),
$$


and

$$
\rho(r) \varphi(x, r)+\sup _{t>r} \rho(t) \varphi(x, t) \leq C \varphi(x, r)^{\frac{p}{q}}
$$

where $C$ does not depend on $x \in \mathbb{R}^{n}$ and $r>0$.

Then the operator $M_{\rho}$ is bounded from $M_{p, \varphi^{\frac{1}{p}}}$ to $W M_{q, \varphi^{\frac{1}{q}}}$ and for $p>1$ from $M_{p, \varphi}$ to $M_{q, \varphi^{\frac{1}{q}}}$.

Proof. Let $x_{0} \in \mathbb{R}^{n}, 1 \leq p<q<\infty$ and $f \in M_{p, \varphi} \frac{1}{p}$. Write $f=f_{1}+f_{2}$, where $B=B(x, r), f_{1}=f \chi_{2 B}$ and $f_{2}=f \chi_{{ }_{(2 B)}}$. Then we have

$$
M_{\rho} f(x) \leq M_{\rho} f_{1}(x)+M_{\rho} f_{2}(x) .
$$

For $M_{\rho} f_{1}(y), y \in B(x, r)$, following Hedberg's trick (see for instance [27], p. 354), we obtain

$$
\begin{aligned}
M_{\rho} f_{1}(y) & =\sup _{t>0} \frac{\rho(t)}{t^{n}} \int_{B(y, t) \cap B(x, 2 r)}|f(z)| d z \\
& \lesssim \sup _{t>0} \int_{B(y, t) \cap B(x, 2 r)} \frac{\rho(|y-z|)}{|y-z|^{n}}|f(z)| d z \\
& \approx \sup _{t>0} \sum_{k=-\infty}^{0} \int_{B(y, t) \cap\left(B\left(x, 2^{k+1} r\right) \backslash B\left(x, 2^{k} r\right)\right)} \frac{\rho(|y-z|)}{|y-z|^{n}}|f(z)| d z \\
& \lesssim \sup _{t>0} \sum_{k=-\infty}^{0} \int_{2^{k} k_{1} r}^{2^{k} k_{2} r} \frac{\rho(s)}{s^{n+1}} d s \int_{B(y, t) \cap B\left(x, 2^{k+1} r\right)}|f(z)| d z \\
& \approx M f(x) \sup _{t>0} \sum_{k=-\infty}^{0} \int_{2^{k} k_{1} r}^{2^{k} k_{2} r} \frac{\rho(s)}{s} d s \\
& =\operatorname{Mf}(x) \int_{0}^{k_{2} r} \frac{\rho(s)}{s} d s \lesssim M f(x) \rho(r) .
\end{aligned}
$$

For $M_{\rho} f_{2}(y), y \in B(x, r)$ from 3.7 we have

$$
\begin{aligned}
M_{\rho} f_{2}(y) & \lesssim \sup _{t>2 r} \frac{\rho(t)}{t^{n}} \int_{B(x, t)}|f(z)| d z \\
& \lesssim \sup _{t>2 r}\|f\|_{L_{p}(B(x, t))} \frac{\rho(t)}{t^{\frac{n}{p}}} .
\end{aligned}
$$

Then from condition (4.2) and inequalities (4.3), 4.4 for all $y \in B(x, r)$ we get

$$
\begin{aligned}
M_{\rho} f(y) & \lesssim \rho(r) M f(x)+\sup _{t>r}\|f\|_{L_{p}(B(x, t))} \frac{\rho(t)}{t^{\frac{n}{p}}} \\
& \leq \rho(r) M f(x)+\|f\|_{M_{p, \varphi} \frac{1}{p}} \sup _{t>r} \varphi(x, t) \rho(t) .
\end{aligned}
$$


Thus, by 4.2 and 4.5 we obtain

$$
\begin{aligned}
& M_{\rho} f(y) \lesssim \min \left\{\varphi(x, t)^{\frac{p}{q}-1} M f(x), \varphi(x, t)^{\frac{p}{q}}\|f\|_{M_{p, \varphi} \frac{1}{p}}\right\} \\
& \lesssim \sup _{s>0} \min \left\{s^{\frac{p}{q}-1} M f(x), s^{\frac{p}{q}}\|f\|_{M_{p, \varphi} \frac{1}{p}}\right\}=(M f(x))^{\frac{p}{q}}\|f\|_{M_{p, \varphi}^{\frac{1}{p}}}^{1-\frac{p}{q}},
\end{aligned}
$$

where we have used that the supremum is achieved when the minimum parts are balanced. Hence for all $y \in B(x, r)$, we have

$$
M_{\rho} f(y) \lesssim(M f(x))^{\frac{p}{q}}\|f\|_{M_{p, \varphi}^{\frac{1}{p}}}^{1-\frac{p}{q}} .
$$

Consequently the statement of the theorem follows in view of the boundedness of the maximal operator $M$ in $M_{p, \varphi^{\frac{1}{p}}}$ provided by Theorem $\mathrm{D}$ in virtue of condition 4.1.

$$
\begin{aligned}
\left\|M_{\rho} f\right\|_{W M_{q, \varphi} \frac{1}{q}} & =\sup _{x \in \mathbb{R}^{n}, t>0} \varphi(x, t)^{-\frac{1}{q}} t^{-\frac{n}{q}}\left\|M_{\rho} f\right\|_{W L_{q}(B(x, t))} \\
& \lesssim\|f\|_{M_{p, \varphi}^{\frac{p}{q}}}^{1-\frac{p}{p}} \sup _{x \in \mathbb{R}^{n}, t>0} \varphi(x, t)^{-\frac{1}{q}} t^{-\frac{n}{q}}\|M f\|_{W L_{p}(B(x, t))}^{\frac{p}{q}} \\
& =\|f\|_{M_{p, \varphi}^{\frac{p}{q}}}^{1-\frac{p}{p}}\left(\sup _{x \in \mathbb{R}^{n}, t>0} \varphi(x, t)^{-\frac{1}{p}} t^{-\frac{n}{p}}\|M f\|_{W L_{p}(B(x, t))}\right)^{\frac{p}{q}} \\
& =\|f\|_{M_{p, \varphi}^{\frac{p}{q}}}^{1-\frac{p}{p}}\|M f\|_{W M_{p, \varphi}^{\frac{1}{q}}}^{\frac{1}{p}} \\
& \lesssim\|f\|_{M_{p, \varphi}^{\frac{1}{p}}},
\end{aligned}
$$

and

$$
\begin{aligned}
\left\|M_{\rho} f\right\|_{M_{q, \varphi} \frac{1}{q}} & =\sup _{x \in \mathbb{R}^{n}, t>0} \varphi(x, t)^{-\frac{1}{q}} t^{-\frac{n}{q}}\left\|M_{\rho} f\right\|_{L_{q}(B(x, t))} \\
& \lesssim\|f\|_{M_{p, \varphi}^{\frac{1}{q}}}^{1-\frac{p}{p}} \sup _{x \in \mathbb{R}^{n}, t>0} \varphi(x, t)^{-\frac{1}{q}} t^{-\frac{n}{q}}\|M f\|_{L_{p}(B(x, t))}^{\frac{p}{q}} \\
& =\|f\|_{M_{p, \varphi}^{\frac{p}{q}}}^{1-\frac{p}{p}}\left(\sup _{x \in \mathbb{R}^{n}, t>0} \varphi(x, t)^{-\frac{1}{p}} t^{-\frac{n}{p}}\|M f\|_{L_{p}(B(x, t))}\right)^{\frac{p}{q}} \\
& =\|f\|_{M_{p, \varphi}^{\frac{1}{q}}}^{1-\frac{p}{p}}\|M f\|_{M_{p, \varphi} \frac{p}{p}}^{\frac{1}{p}} \\
& \lesssim\|f\|_{M_{p, \varphi} \frac{1}{p}},
\end{aligned}
$$

if $1<p<q<\infty$.

In the case $\rho(t)=t^{\alpha}$ from Theorem 4.2 we get the Adams type result on generalized Morrey spaces (see [16, Theorem 5.7, p. 182]).

In the case $\rho(t)=t^{\alpha}, \varphi(x, t)=t^{\lambda-n}, 0<\lambda<n$ from Theorem 4.2 we get the following Adams's result for the fractional maximal operator. 
Corollary 4.4. Let $0<\alpha<n, 1<p<\frac{n}{\alpha}, 0<\lambda<n-\alpha p$ and $\frac{1}{p}-\frac{1}{q}=\frac{\alpha}{n-\lambda}$. Then for $p>1$, the operator $M_{\alpha}$ is bounded from $M_{p, \lambda}$ to $M_{q, \lambda}$ and for $p=1, M_{\alpha}$ is bounded from $M_{1, \lambda}$ to $W M_{q, \lambda}$.

Remark 4.3. Note that, the condition (3.1) is weaker than the following condition which was given in [17] for $I_{\rho}$ :

$$
\int_{1}^{\infty} \frac{\rho(t)}{t^{n}} \frac{d t}{t}<\infty .
$$

For example, the function

$$
\rho(t)=\frac{t^{n}}{\log (e+t)}, t>0
$$

satisfies (3.1), but not 4.6). This example shows that the function $\rho$ satisfies Theorems 3.1 and 4.2, but does not satisfy the assumptions of Theorems 16 and 22 in [17. In other words, the condition (3.1) which satisfies our main theorems, is better (more general and comprehensive) than the condition (4.8) which satisfies the main theorems were given in [17].

\section{REFERENCES}

[1] Adams, D.R., A note on Riesz potentials, Duke Math. 42 (1975) 765-778.

[2] Akbulut, A., Guliyev, V.S. and Mustafayev, R., On the boundedness of the maximal operator and singular integral operators in generalized Morrey spaces, Math. Bohem. 137 (1) (2012), $27-43$.

[3] Burenkov, V., Guliyev, H.V. and Guliyev, V.S., Necessary and sufficient conditions for boundedness of the Riesz operator in the local Morrey-type spaces, Doklady Ross. Akad. Nauk. Matematika 412 (5) (2007), 585-589 (Russian). English trans. in Acad. Sci. Dokl. Math. 76 (2007).

[4] Burenkov, V. and Guliyev, V.S., Necessary and sufficient conditions for the boundedness of the Riesz operator in local Morrey-type spaces, Potential Anal. 30 (2009), no. 3, 211-249.

[5] Burenkov, V., Guliyev, H.V. and Guliyev, V.S. Necessary and sufficient conditions for boundedness of the fractional maximal operator in the local Morrey-type spaces, Doklady Ross. Akad. Nauk. Matematika 409 (4) (2006), 443-447 (Russian). English trans. in Acad. Sci. Dokl. Math. 74 (2006).

[6] Burenkov, V., Guliyev, H.V. and Guliyev, V.S., Necessary and sufficient conditions for boundedness of the fractional maximal operator in the local Morrey-type spaces, J. Comput. Appl. Math. 208 (2007), no. 1, 280-301.

[7] Burenkov, V., Gogatishvili, A., Guliyev, V. and Mustafayev, R.Ch., Boundedness of the Riesz potential in local Morrey-type spaces, Potential Anal. 35 (2011), no. 1, 67-87.

[8] Burenkov, V., Gogatishvili, A., Guliyev, V.S. and Mustafayev, R., Boundedness of the fractional maximal operator in local Morrey-type spaces, Complex Var. Elliptic Equ. 55 (8-10) (2010), 739-758.

[9] Chiarenza, F. and Frasca, M., Morrey spaces and Hardy-Littlewood maximal function, Rend Mat. 7 (1987), 273-279.

[10] Eridani, A., On the boundedness of a generalized fractional integral on generalized Morrey spaces, Tamkang J. Math. 33 (2002), no. 4, 335-340. 
[11] Eridani, A., Gunawan, H., Nakai, E. and Sawano, Y., Characterizations for the generalized fractional integral operators on Morrey spaces, Math. Inequal. Appl. 17 (2014), no. 2, 761-777.

[12] Eridani, A., Gunawan, H. and Nakai, E., On generalized fractional integral operators, Sci. Math. Jpn. 60 (2004), no. 3, 539-550.

[13] Guliyev, V.S., Integral operators on function spaces on the homogeneous groups and on domains in $R^{n}$. Doctoral degree dissertation, Mat. Inst. Steklov, Moscow, 1994, 329 pp. (in Russian)

[14] Guliyev, V.S., Boundedness of the maximal, potential and singular operators in the generalized Morrey spaces, J. Inequal. Appl. 2009, Art. ID 503948, 20 pp.

[15] Guliyev, V.S., Generalized local Morrey spaces and fractional integral operators with rough kernel, J. Math. Sci. (N.Y.) 193 (2) (2013), 211-227.

[16] Guliyev, V.S. and Shukurov, P.S., On the boundedness of the fractional maximal operator, Riesz potential and their commutators in generalized Morrey spaces. Advances in Harmonic Analysis and Operator Theory, Series: Operator Theory: Advances and Applications, 229 (2013), 175-199.

[17] Guliyev, V.S., Ismayilova, A.F., Kucukaslan, A. and Serbetci, A., Generalized fractional integral operators on generalized local Morrey spaces, Journal of Function Spaces, Volume 2015, Article ID 594323, 8 pages.

[18] Gunawan, H., A note on the generalized fractional integral operators, J. Indones. Math. Soc. 9 (2003), no. 1, 39-43.

[19] Kucukaslan, A., Hasanov, S.G. and Aykol, C., Generalized fractional integral operators on vanishing generalized local Morrey spaces, International Journal of Mathematical Analysis, 11, 2017, no. 6, $277-291$.

[20] Mizuhara, T., Boundedness of some classical operators on generalized Morrey spaces, Harmonic Analysis (S. Igari, Editor), ICM 90 Satellite Proceedings, Springer - Verlag, Tokyo (1991) 183-189.

[21] Morrey, C.B., On the solutions of quasi-linear elliptic partial differential equations, Trans. Amer. Math. Soc. 43 (1938) 126-166.

[22] Nakai, E., Hardy-Littlewood maximal operator, singular integral operators and Riesz potentials on generalized Morrey spaces, Math. Nachr. 166 (1994) 95-103.

[23] Nakai, E., On generalized fractional integrals, Taiwanese J. Math. 5 (2001), 587-602.

[24] Peetre, J., On the theory of $M_{p, \lambda}$, J. Funct. Anal. 4 (1969) 71-87.

[25] Sawano, Y., Sugano, S. and Tanaka, H., Generalized fractional integral operators and fractional maximal operators in the framework of Morrey spaces, Trans. Amer. Math. Soc. 363 (2011), no. 12, 6481-6503.

[26] Sawano, Y., Sugano, S. and Tanaka, H., Orlicz-Morrey spaces and fractional operators, Potential Anal., 36 (4) (2012), 517-556.

[27] Stein, E.M., Harmonic Analysis: Real Variable Methods, Orthogonality and Oscillatory Integrals, Princeton Univ. Press, Princeton NJ, 1993. 
Current address: A. Kucukaslan(Coressponding author): Institute of Mathematics, Czech Academy of Sciences, 11567, Prague, Czech Republic, School of Applied Sciences, Pamukkale University, 20680, Denizli, Turkey

E-mail address: kucukaslan@pau.edu.tr

ORCID Address: http://orcid.org/0000-0002-9207-8977

Current address: V.S. Guliyev: Nikolskii Institute of Mathematics at RUDN University, 117198, Moscow, Russia, Department of Mathematics, Dumlupinar University, 43100, Kutahya, Turkey

E-mail address: vagif@guliyev.com

ORCID Address: http://orcid.org/0000-0001-7486-0298

Current address: A. Serbetci: Ankara University, Faculty of Sciences, Dept. of Mathematics, Ankara, TURKEY, Department of Mathematics, Cankiri Karatekin University, Cankiri, Turkey

E-mail address: serbetci@ankara.edu.tr

ORCID Address: http://orcid.org/0000-0001-6362-7044 